\title{
Transcriptome analysis of the Baikal whitefish head kidney (Coregonus sp., Coregonidae)
}

\author{
Tuyana Sidorova \\ Laboratory of Ichtiology \\ Limnological Institute, Siberian Branch \\ of the Russian Academy of Sciences \\ Irkutsk, Russia \\ tuyana_be@mail.ru \\ Lubov Sukhanova \\ Laboratory of Ichtiology \\ Limnological Institute, Siberian Branch \\ of the Russian Academy of Sciences \\ Irkutsk, Russia \\ 1sukhanova@yandex.ru
}

\author{
Ivan Kutyrev \\ Laboratory of Parasitology and \\ Ecology of Aquatic Organisms \\ Institute of General and Experimental \\ Biology, Siberian Branch of the \\ Russian Academy of Sciences \\ Ulan-Ude; Institute of Biology, Irkutsk \\ State University, Irkutsk Russia \\ ikutyrev@yandex.ru \\ Olga Mazur \\ Laboratory of Parasitology and \\ Ecology of Aquatic Organisms \\ Institute of General and Experimental \\ Biology, Siberian Branch of the \\ Russian Academy of Sciences \\ Ulan-Ude, Country \\ olmaz33@yandex.ru
}

\author{
Kirill Khabudaev \\ Department of Cell Ultrastructure \\ Limnological Institute, Siberian Branch \\ of the Russian Academy of Sciences \\ Irkutsk, Russia \\ onexkirill@gmail.com
}

\begin{abstract}
Tapeworms of the genus Dibothriocephalus are widely distributed all around the world, with some being agents of human diphyllobothriasis, one of the most important fishborne zoonosis caused by a cestode parasite. The study of immunological relations between plerocercoids of Dibothriocephalus dendriticus and its salmonid fish hosts is of great importance. Hereby, we carried out complete transcriptome analyses of whitefish Coregonus baicalensis head kidney. Using Blast2Go, we revealed transcripts distribution among categories: biological processes, molecular functions.
\end{abstract}

\section{Keywords - whitefish, head kidney, transcriptome}

\section{Introduction}

Coevolution between hosts and their parasites is predicted to be dynamic and rapid, mainly because fitness costs caused by parasites are high, parasites are ubiquitous, and they often evolve rapidly. The majority of parasites have evolved strategies to evade the immune response of their hosts. Tapeworms of the genus Dibothriocephalus are widely distributed all around the world, with some being agents of human diphyllobothriasis, one of the most important fishborne zoonosis caused by a cestode parasite. Humans can contract this parasite by eating raw or partially cooked fish containing Dibothriocephalus plerocercoids [1]. We began the study of immunological relations between plerocercoids of Dibothriocephalus dendriticus and its salmonid fish hosts. The aim of this study was to conduct transcriptome analysis of the whitefish Coregonus baicalensis head kidney.

\section{Material and methods}

Samples of whitefish head kidneys were homogenized in liquid nitrogen and RNA was extracted using the RNEasy Mini (Qiagen) kit, according to manufacturer's instructions. Complementary DNA (cDNA) libraries were then prepared using 250-500 ng of total RNA. Illumina sequencing adapters were then ligated to the cDNA, which were then PCR amplified for 15 cycles of $98^{\circ} \mathrm{C} 10$ second denaturing, $60{ }^{\circ} \mathrm{C}$ 30 second annealing and $72^{\circ} \mathrm{C} 30$ second extension and using a final extension step of $72{ }^{\circ} \mathrm{C}$ for 5 minutes. The libraries were then used for clusters generation Illumina NextSeq550 and NextSeq 550 High Output v2 Kit (300 cycles) chemistry. High quality reads were merged and assembled using Trinity (with default parameters). Open reading frames were predicted using Transdecoder (http://transdecoder.sf.net) with default parameters. Conserved domains and associated Gene Ontology (GO) annotation [2] were predicted using Blast2Go.

\section{Results and discussion}

For the first time, complete transcriptome analyses of Baikal whitefish $C$. baicalensis head kidney was done. In total, 90253643 Illumina paired-end reads were generated from the cDNA libraries. Using Blast2Go, we revealed transcripts distribution among categories: biological

Fig. 1. Gene Ontology annotations. Classification of the C. lavaretus head kidney proteins in terms of molecular functions (A), biological processes (B), and cellular components (C).

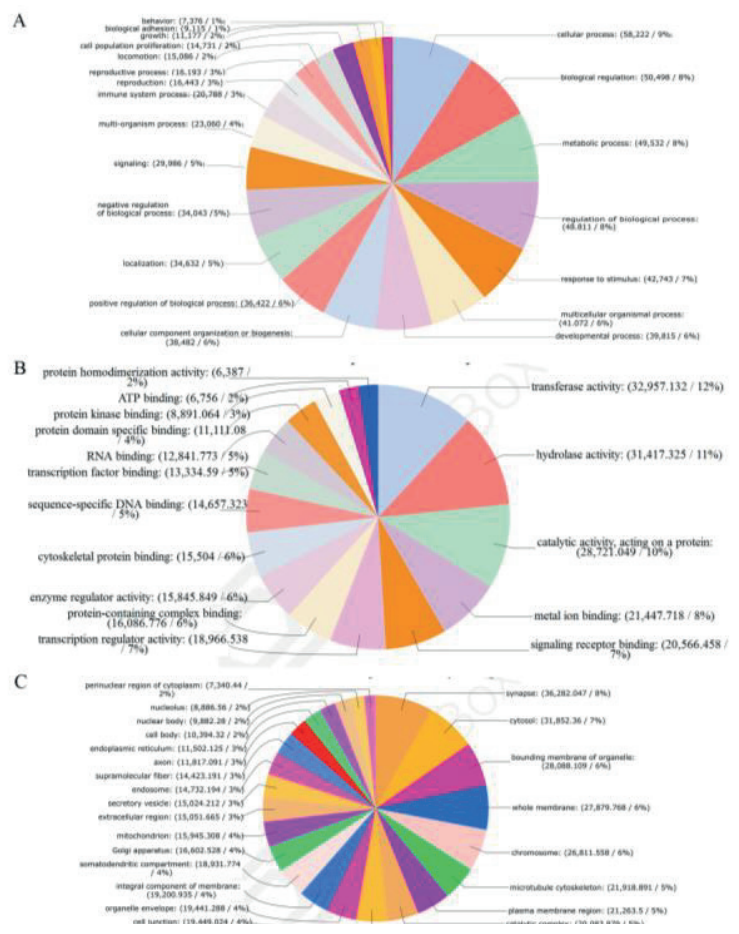


processes, molecular functions (Fig. 1). For biological processes predominant transcripts were: cellular processes $(9 \%)$, biological regulation $(8 \%)$, metabolic processes $(8 \%)$, and regulation of biological processes (8\%). Among molecular functions the next transcripts prevailed: transferase activity (12\%), hydrolase activity (11\%), catalytic activity, acting on protein $(10 \%)$. Synapse $(8 \%)$ cytosol $(7 \%)$, bounding membrane of organelle $(6 \%)$, whole membrane $(6 \%)$, and chromosome $(6 \%)$ were predominant transcripts in cellular components category.

Obtained transcriptome of $C$. baicalensis head kidney will be used further to search immunocompetent genes involved in immune response against helminth invasion. Obtained information on the genes will be necessary for study of cellular and molecular relations between cestode plerocercoids and fish host immune system using real-time polymerase chain reaction, flow cytometry or confocal microscopy.

\section{ACKNOWLEDGMENT}

This study was performed under the theme of Russian government task (government registration No. AAAA-A17117011810039-4) and funded by grant of the Russian Foundation of Fundamental Researches (grant No. 18-3420015) and the Ministry of Science and Higher Education of the Russian Federation (project No. FZZE-2020-0026; I.A.K.)

\section{REFERENCES}

[1] J. Dupouy-Camet, H. Year, Diphyllobothrium, in: D. Liu (Ed.), Molecular detection of foodborne pathogens, Taylor and Francis, London and New York, 2010, pp. 781-788.

[2] Ashburner M, Ball CA, Blake JA, Botstein D, Butler H, Cherry JM, et al. Geneontology: tool for the unification of biology. The Gene Ontology Consortium. NatGenet 2000;25:25-9. doi:10.1038/75556. 